\title{
Razones del incumplimiento del esquema básico de vacunación en una comunidad rural de Aguascalientes
}

\author{
Muñoz-Trinidad Jessica*, Villalobos-Navarro Andrea*, Gómez-Chávez Juan Ricardo*, De Loera- \\ Díaz Indira Nataly*, Nieto-Aguilar Andrea*, Macías-Galaviz Ma. Teresa**
}

\begin{tabular}{|c|c|}
\hline $\begin{array}{l}\text { Resumen } \\
\text { Introducción: México tiene uno de los esquemas de vacunación } \\
\text { más completos en América Latina y su éxito en nuestro país es } \\
\text { innegable. Actualmente el incumplimiento del esquema básico de } \\
\text { vacunación es un grave problema que tiene como consecuencia } \\
\text { un incremento en la incidencia de las enfermedades prevenibles } \\
\text { por vacunación, las cuales repercuten en el aumento del índice } \\
\text { de morbimortalidad en menores de } 5 \text { años; el objetivo del estudio } \\
\text { es develar las razones del incumplimiento del esquema básico } \\
\text { de vacunación en una comunidad rural de Aguascalientes. Me- } \\
\text { todología: estudio de enfoque cualitativo con base en la teoría } \\
\text { fundamentada, realizado en una comunidad rural de Aguasca- } \\
\text { lientes durante el periodo septiembre-diciembre del año } 2019 \text {; } \\
\text { mediante una entrevista semiestructurada a madres de niños con } \\
\text { esquemas básicos de vacunación incompletos, llegando a la sa- } \\
\text { turación teórica con II de ellas. Resultados: se identificaron siete } \\
\text { categorías que influyen en el incumplimiento de los esquemas de } \\
\text { vacunación: razones propias de la madre, del menor, culturales, } \\
\text { geográficas, económicas, institucionales y del personal de salud. } \\
\text { Conclusión: las razones expresadas por las madres fueron diver- } \\
\text { sas y muchas de estas referidas en estudios anteriores. Lo que re- } \\
\text { salta en esta investigación y la principal razón identificada fue el } \\
\text { desinterés para su cumplimiento. El incumplimiento del esquema } \\
\text { básico de vacunación es un fenómeno multifactorial en donde la } \\
\text { educación para la salud es un tema indispensable para su reso- } \\
\text { lución; siendo una comunidad rural, su población se vuelve más } \\
\text { vulnerable, por lo que se debe intervenir en las razones identifica- } \\
\text { das. LuxméDica año 16, Número 47, mayo-AGosto 2021, PP 13-22. }\end{array}$ & 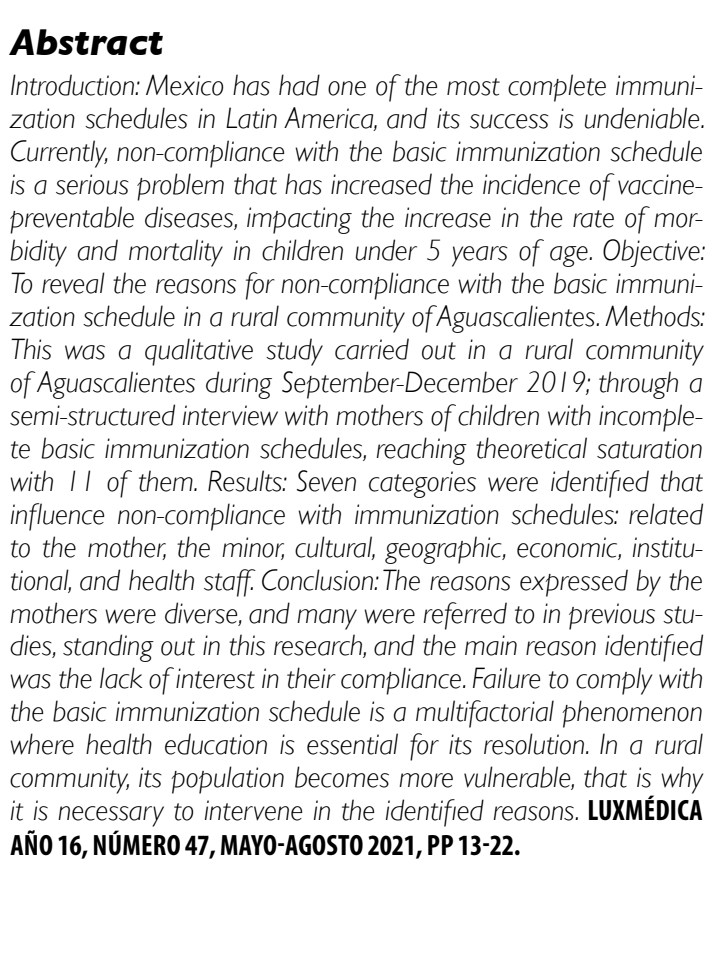 \\
\hline $\begin{array}{r}\text { Palabras clave: vacunación, esquema de vacunación, } \\
\text { comunidad rural. }\end{array}$ & $\begin{array}{l}\text { Keywords: immunization, immunization schedule, rural } \\
\text { community }\end{array}$ \\
\hline
\end{tabular}

\footnotetext{
Estudiante de la Licenciatura en Enfermería del Centro de Ciencias de la Salud de la Universidad Autónoma de Aguascalientes. https://orcid.org/0000-0003-3531-7406 Correo electrónicoal203773@edu.uaa.mx

Estudiante de la Licenciatura en Enfermería del Centro de Ciencias de la Salud de la Universidad Autónoma de Aguascalientes. https://orcidorg/0000-0003-4335-2244 Correo electrónico andrea.compbas@hotmailcom

Estudiante de la Licenciatura en Enfermería del Centro de Ciencias de la Salud de la Universidad Autónoma de Aguascalientes. https://orcid.org/0000-0003-3624-4552 Correo electrónico jurigocha@gmailcom

Estudiante de la Licenciatura en Enfermería del Centro de Ciencias de la Salud de la Universidad Autónoma de Aguascalientes. https://orcid.org/0000-0003-3563-5594 Correo electrónico indiradeloera@gmail.com

Estudiante de la Licenciatura en Enfermería del Centro de Ciencias de la Salud de la Universidad Autónoma de Aguascalientes. https://orcid.org/0000-0002-2209-9131 Correo electrónico andreanietto.18@hotmail.com

Profesora del Departamento de Enfermería del Centro de Ciencias de la Salud de la Universidad Autónoma de Aguascalientes. https://orcid.org/0000-0002-7600-7626 Correo electrónico tmg2699@hotmail.com

Fecha de recibido: 28 de octubre de 2020 Fecha de aceptación: 19 de febrero 2021

Correspondencia: MSPS Ma. Teresa Macías Galaviz, Departamento de Enfermería, edificio 105, Centro de Ciencias de la Salud. Ciudad Universitaria. Universidad Autónoma de Aguascalientes, Avenida Universidad 940. Código postal 20131. Teléfono 9108437. Aguascalientes, Aguascalientes, México. Correo electrónico: jurigocha@gmail.com
} 


\section{Introducción}

Los primeros años de un niño o una niña son trascendentales para su formación ${ }^{1}$ ya que durante ese periodo se sientan las bases de su desarrollo físico, motor y socioemocional. Por ello, en esta etapa es fundamental garantizarles las mejores condiciones de salud, nutrición, protección y cuidados para que tengan todo lo necesario para un buen comienzo. ${ }^{2}$ Actualmente se tienen los conocimientos y la posibilidad de mejorar en gran medida su salud y bienestar, de realizar el cambio transformador necesario para configurar un futuro más próspero y sostenible. ${ }^{3}$

La prevención y la promoción de la salud constituyen un eje fundamental de la salud pública; por ello, son componentes esenciales del modelo de atención a la salud en México. ${ }^{4}$

La vacunación es una estrategia primordial para mantener a los infantes saludables, y protegidos de enfermedades infecciosas prevenibles. Actualmente, México dispone de un sistema de vacunación compuesto por 13 biológicos para niños y niñas menores de ocho años, que protege a esta población contra 15 enfermedades prevenibles; ${ }^{5}$ tomando en cuenta lo anterior, se entiende por esquema básico de vacunación a aquel orientado a la aplicación de las vacunas establecidas en los lineamientos emitidos por la Secretaría de Salud a menores de 5 años de edad. ${ }^{6}$ La omisión de al menos una dosis registrada en la cartilla nacional de vacunación se considera esquema incompleto. ${ }^{7}$

En los últimos cinco años ha existido un progreso favorable en los porcentajes de cobertura de vacunación del esquema completo, como resultado del Programa de Vacunación Universal que lleva adelante el gobierno federal. ${ }^{5}$

Los registros del Centro Nacional de Salud para la Infancia y la Adolescencia (CENSIA) muestran que México alcanzó en el 2014 la meta establecida de $90 \%$ de cobertura de vacunación del esquema completo en niños menores de un año de edad. ${ }^{5}$ La Encuesta nacional de niños, niñas y mujeres (ENIM, 2015) y la Encuesta nacional de salud y nutrición (ENSANUT, 2012) proporcionan un panorama diferente al de los registros del CENSIA, ya que cuentan con estadísticas de vacunación basadas en la observación directa de los registros de la Cartilla Nacional de Vacunación. ${ }^{5,8}$ Los resultados de cobertura de vacunación de ambas encuestas señalan que, a diferencia de los registros del CENSIA, en México aún hay una proporción importante de estos menores que no están adecuadamente vacunados siendo vulnerables ante enfermedades prevenibles, debatiendo la congruencia del oficialismo contra la realidad. ${ }^{5}$

Autores como Escobar Díaz y colaboradores (2017), en su trabajo titulado Motivos de no vacunación en menores de cinco años en cuatro Ciudades Colombianas, identificaron diferentes factores que pueden influir en el incumplimiento de los esquemas de vacunación, como el temor a la reacción posvacunal, las condiciones socioeconómicas, geográficas y de seguridad de la población, 
las condiciones laborales del personal de vacunación, los problemas administrativos y económicos y el desarrollo precario de los sistemas de información. ${ }^{9}$ Mientras que Escobedo Collado y Portocarrero Ramos (2018), en su trabajo Factores socioculturales e institucionales relacionados con el incumplimiento del esquema de vacunación en madres de menores de 13 meses. P.S. Peruarbo, Arequipa-2017, concluyeron que los factores socioculturales como la edad de la madre, vivienda y creencias respecto a la vacuna tienen relación significativa con el incumplimiento del esquema de vacunación. ${ }^{10}$

Actualmente el incumplimiento del esquema de vacunación es un grave problema que tiene como consecuencia un incremento en la incidencia de las enfermedades prevenibles por vacunación, las cuales podrían repercutir en el aumento del índice de morbimortalidad en menores de 5 años. ${ }^{11}$

De acuerdo con el Consejo Nacional de Evaluación de la Política de Desarrollo Social (CONEVAL), una comunidad rural se compone por aquellas localidades que cuentan con menos de 2 mil 500 habitantes. ${ }^{12}$ Actualmente, persisten las grandes diferencias entre grupos urbanos y rurales, así como entre hispano-parlantes e indígenas. ${ }^{13}$ La ubicación geográfica de las comunidades rurales se relaciona con su grado de marginación por lo que las personas que viven en asentamientos dispersos y aislados enfrentan mayores rezagos sociales. ${ }^{14}$ Estimaciones de la Encuesta Nacional de Ocupación y Empleo (ENOE) 2018, en México existen 11.4 millones de niñas y niños de cinco y menos años, y el $27.3 \%$ de esta población habita en zonas rurales. ${ }^{15}$ Así, factores como vivir en situación de pobreza o en una comunidad rural alejada sin acceso a servicios básicos provoca que algunas de las familias más pobres no tengan la posibilidad de afrontar los costos de trasladarse a cabeceras municipales o comunidades más pobladas donde pueden acceder a éstos, poniendo en desventaja a millones de niños y niñas en México. ${ }^{16}$

Es importante resaltar que la comunidad cuenta con una cultura y hábitos determinados por una serie de redes sociales y grupos de liderazgo específicos, que muchas veces son desconocidos por los servicios de salud. La articulación con ellos puede ser crucial para un desempeño eficiente de los servicios de salud. Contar con una estrategia desde los servicios de salud es importante para identificar la cultura local, las interrelaciones comunitarias, los vínculos y redes sociales, los diferentes actores, sus expectativas y necesidades, así como una estrategia para su vinculación con la red de servicios y fortalecer las redes sociales comunitarias. ${ }^{17}$

Contar con información sobre las condiciones que favorecen o perjudican la salud, es importante para focalizar la atención hacia los aspectos por trabajar en cada individuo y familia. ${ }^{18}$ Considerando lo anterior, surgió la inquietud de explorar el tema desde el enfoque cualitativo aplicando la Teoría Fundamentada en Datos, ${ }^{19}$ 
con el objetivo de develar las razones del incumplimiento del esquema básico de vacunación en una comunidad rural de Aguascalientes, mediante la identificación y el análisis de los factores que intervienen en este fenómeno, para finalmente describir y clasificar los datos expresados por las madres.

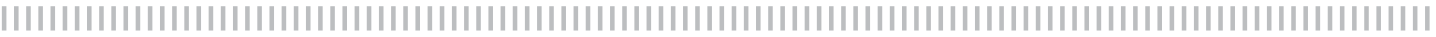

\section{Material y métodos}

Se realizó un estudio de tipo cualitativo, exploratorio con base en la Teoría Fundamentada en Datos, en la comunidad rural Ojo de Agua del Mezquite, San Francisco de los Romo, Aguascalientes, durante el periodo septiembre-diciembre del año 2019. Se llevó a cabo una entrevista semiestructurada a madres con hijos menores de cinco años que incumplían con el esquema básico de vacunación y que pertenecían a la comunidad. Se realizó un muestreo teórico en función de unidades de análisis, las cuales se determinaron antes y durante la inmersión inicial, hasta que se alcanzó la saturación teórica con 11 entrevistas.

Se gestionó con el líder comunitario el préstamo de un salón, el cual fue acondicionado para la realización de las entrevistas, asegurando un ambiente apto y cómodo para las participantes, a las cuales se les solicitó autorización para colocar una grabadora de audio a su costado evitando así barreras físicas de comunicación o interferencias de sonido.
Con apego al Reglamento de la Ley General de Salud en Materia de Investigación, este estudio fue considerado sin riesgo, ya que no se realizó ninguna intervención o modificación intencionada de las variables biopsicosociales de las participantes. Se proporcionó y revisó junto con cada participante un consentimiento informado que posteriormente fue firmado. ${ }^{20}$

El análisis se realizó por medio de la lectura de las transcripciones con apoyo del software Listen $N$ write y de la simbología de Jefferson, codificación, visualización, reducción e interpretación de datos con el apoyo del software ATLAS. ti. ${ }^{21}$

Para garantizar el rigor metodológico, se usaron los criterios de credibilidad (transcripción textual de manera exacta, resguardo de notas de campo y triangulación), transferibilidad (recogida de datos hasta la saturación teórica) y auditabilidad (mediante la descripción de las características de los informantes, mecanismos de grabación y trascripción fiel).

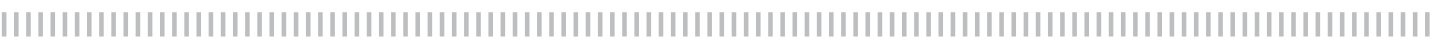

\section{Resultados}

La edad de las participantes oscilaba entre 20-40 años, cinco de ellas casadas y seis en unión libre, con ocupación predominante al hogar; dos contaban con escolaridad de primaria y nueve de secundaria; seis de ellas no disponían con medio de transporte propio.

Se obtuvieron 163 segmentos, de los cuales se codificaron en siete categorías y 20 códigos (tabla 1 ). 


\section{Tabla I}

\section{Categorías de análisis}

\begin{tabular}{|l|l|c|}
\hline CATEGORÍAS & CÓDIGOS & NÚMERO DE SEGMENTOS \\
\hline PROPIAS DE LA MADRE & Déficit de conocimiento & 10 \\
\hline & Desconfianza & 1 \\
\hline & Desinterés & 28 \\
\hline & Experiencia-Vivencia & 12 \\
\hline & Falta de tiempo & 1 \\
\hline & Inexperiencia en el rol parental & 1 \\
\hline & Número de hijos & 7 \\
\hline CULTURALES & Temor & 4 \\
\hline & Creencias individuales & 12 \\
\hline PROPIAS DEL MENOR & Conciencia colectiva & 4 \\
\hline \multirow{2}{*}{ GEOGRÁFICAS } & ESAVI & 6 \\
\hline & Estado de salud & 2 \\
\hline PERSONAL DE SALUD & Distancia & 13 \\
\hline \multirow{2}{*}{ ECONÓMICAS } & Transporte & 19 \\
\hline INSTITUCIONALES & Actitud del personal de enfermería & 5 \\
\hline & Actitud del personal médico & 2 \\
\hline TOTAL & Falta de recurso económico & 16 \\
\hline & Falta de biológico & 6 \\
\hline & Horarios insuficientes & 6 \\
\hline & Oportunidades perdidas & 163 \\
\hline
\end{tabular}

Fuente: entrevista semiestructurada, comunidad Ojo de Agua del Mezquite, septiembre-diciembre 2019.

\section{Razones propias de la madre}

Se consideraron todos aquellos factores relacionados con la madre del menor que intervinieron en el incumplimiento del esquema básico de vacunación, tales como el déficit de conocimiento de la importancia de la vacunación, la desconfianza expresada ante la atención del profesional de salud, el desinterés para llevar a cabo dicha acción, experiencia-vivencia, falta de tiempo, inexperiencia en el rol parental, número de hijos, y el temor a las reacciones posvacunales, tal como lo manifiestan en los siguientes párrafos:

[Desinterés] (...) "pos a veces por falta de no salir o cuando vienen aquí, ya sería por flojera o por no dejar de hacer lo que estoy haciendo por ir a vacunarlo, verdad. Pues, que hay muchas personas que asíle hacen, a después los llevo allá, ahorita toy ocupada” ...VAS

[Experiencia-vivencia] (...) “un tío mío apenas tiene poquito que murió de eso, le pusieron esa vacuna y falleció. Pos uno agarra el miedo a la vacuna” ... YLM

\section{Razones culturales}

Se consideró todos aquellos factores relacionados con los aspectos sociales y culturales de la comunidad que intervinieron en el incumplimiento del esquema básico de vacunación, tales como las creencias individuales que reflejan las percepciones pro- 
pias de la madre y la conciencia colectiva entendida como las creencias compartidas y acciones morales de la comunidad, tal como lo enuncian en los siguientes párrafos:

[Creencias individuales] (...) "yo no me las he aplicado y pues yo digo pues a mí nunca me ha pasado nada y yo digo a los niños pues tampoco se las pongo" ... LIMC

[Conciencia colectiva] (...) "Pues he escuchado: mm personas que se dicen que las vacunas no sirven $(x)$ que al contrario se enferma más" ...MLM

\section{Razones propias del menor}

Se integraron todas aquellas razones o situaciones relacionadas con el menor de cinco años por las cuales no fue vacunado en tiempo y forma de acuerdo con lo establecido en los lineamientos emitidos por la Secretaría de Salud, tales como los Eventos Supuestamente Atribuidos a la Vacunación o Inmunización (ESAVI) y estado de salud; tal como lo expresan los siguientes discursos:

[ESAVI] (...) "Por tal de no batallar porque como con los otros les daba calentura y sabe cuánto, ay yo decía, no, yo no lo llevo a vacunar y lo que los tiene uno que bañar y luego están bien chillones y sabe cuánto" ...MASC

[Estado de salud] (...) "Ah sí se me ponía: se me puso malita y duró tiempo. Porque se enfermó de los bronquios. Entonces como que estaba recayendo, me decían "no hasta que se alivie y no porque le daba calentura" o así me decían no. Entonces, ya después que me la apliqué, yo dije, no se la pongo, ¿por qué? porque a poco recae y yo también dije y no se la he puesto”... MLM

\section{Razones geográficas}

Todos aquellos factores relacionados con las características del territorio que habita la población de estudio que repercutieron en el incumplimiento del esquema básico de vacunación, tales como la distancia que existe entre la ubicación de la comunidad hasta la unidad de salud a la que pertenecen, así como la falta de transporte para su traslado, como lo denotan los siguientes segmentos:

[Distancia] (...) “a veces no hay, pues como quién te mueva o que no tiene en qué moverse. Que a lo mejor si sí se hace y poquito más retirao, que a lo mejor digan, no es que se llegó la fecha y no, no tuve quién me moviera o no hubo quién me llevara, y a veces por eso uno deja pasar la fecha” ...MLM

[Transporte] (...) "Si a veces no podía porque no tenía en qué ir o a veces no podía porque no tenía, o sea, a veces también una cosa es que no tengo en qué ir y a veces no tengo quién me lleve, porque a veces trabaja mi pareja y pos a veces me llevaba mi suegra, a veces ella trabaja” ...MAV

\section{Razones del personal de salud}

Se consideraron todos los factores relacionados con la actitud y el ejercicio profesional del personal de enfermería y médico encargado del servicio de vacunación, los cuales repercutieron en el incumplimiento del esquema básico de vacunación; un ejemplo de ellos son los fragmentos de entrevistas descritos a continuación:

[Actitud del personal médico] (...) "Una doctora fue la que nos dijo que ella tampoco se la ponía por lo mismo de que la enfermaba más”... LIMC 
[Actitud del personal de enfermería] (...) "los enfermeros tengan su forma de atendernos, pos si, bueno para mí eso es más que nada que casi uno vaya al corriente con las vacunas, porque no me gusta su forma de ser, su forma de vacunarlos, su forma de agarrarlos $\mathrm{mm}$ no, lo regañan a uno y sabe cuánto le dicen a uno" ... MASC

\section{Razones económicas}

Se consideraron los factores relacionados con el ingreso monetario familiar que disminuyen la posibilidad de llevar a cabo el acto de vacunación, tal como la falta de recurso económico, por lo que se enuncia a continuación:

[Falta de recurso económico] (...) "a veces anda mal económicamente uno, que llega el mero día, que se cumplió sus dos meses y tiene que llevarlo uno a tiempo, chin, pero si no puede porque no me quedó dinero como pa echar vuelta $\mathrm{mm}$, pos pa la siguiente semana, o cuando más o menos nos acomodemos, entonces en ese tiempo a veces sí, si se junta una y se junta la otra, entonces cuando a veces sé, de repente nos llaman la atención... SAN$D R A$

\section{Razones institucionales}

Todas aquellas características administrativas, funcionales y de organización que definen a cada institución y que desfavorecie- ron al servicio de vacunación como la falta de biológico, los horarios insuficientes como lo es la poca disponibilidad del servicio y las oportunidades perdidas de vacunación, por las que, a pesar de no existir contraindicaciones, el menor de cinco años no recibe todas las vacunas necesarias, tal como lo manifiestan los siguientes segmentos:

[Falta de biológico] (...) "la llevé y me dijeron que no la tenían, que ellos me avisaban cuando llegara esa vacuna, entonces incluso que estuvieron nuevamente vacunando yo la llevé, entonces me dijeron lo mismo, que no había ya vacunas que después me volvían a avisar y es por eso que es la causa de que no se la he puesto. Porque me dicen lo mismo de que no, será que no alcanzo, o no la (inaudible) y es por esa causa porque incluso tiene todas menos esa la que le falta”... MLM

[Oportunidades perdidas] (...) "me decían que yo la tenía que llevar dónde, pues sí, donde ella tenía su control de lo de las vacunas y de hecho yo una vez sí le dije a la enfermera le dije, pero- pos si supuestamente quieren llenar los esquemas, pos la vacuna tienen que ponérsela donde sea el centro de salud, aunque la bebé haiga nacido en Aguascalientes. Yo digo que tiene que ser donde sea, el chiste es que tenga su esquema lleno de las vacunas, ya después ya no regresé ahí... SMP

\section{| | | | | | | | | | | | | | | | | | | | | | | | | | | | | | | | | | | | | | | | | | | | | | | | | | | | | | | | | | | | | | | | | | | | | | | | | | | | | | | | | | | | | | | | | | | | | | | | |}

\section{Discusión}

Este estudio identificó diferentes razones de incumplimiento del esquema básico de vacunación.

En razones propias de la madre se identificó el código "temor", razón que también refiere Escobar Diaz y cols., donde se expresaron razones que estaban relacionadas con temor a la reacción posvacunal. Encontraron también que las condiciones socioeco- nómicas y geográficas influyen de manera relevante con el fenómeno en estudio, descritas en esta investigación como razones "geográficas y económicas". 9

El conocimiento es la base para una buena práctica encaminada a la protección de la salud. El déficit de conocimientos puede generar que en ocasiones no exista conciencia del potencial de salud y de los riesgos relacionados con éste, 
conduciendo a la toma de decisiones sin la información necesaria o apropiada que afectan el bienestar; siendo así, Escobedo Collado y cols. (2018), refieren que una de las principales causas del incumplimiento es el nivel de conocimientos sobre vacunas malo, por lo que se coincide, identificando el "déficit de conocimiento". También concluyeron que los factores socioculturales como las creencias respecto a la vacuna, tienen relación significativa con el incumplimiento del esquema de vacunación, coincidiendo con el código "creencias individuales" de la categoría "razones culturales"..$^{10}$ Las creencias se definen como la relación percibida entre un objeto y un atributo, son el elemento cognoscitivo de la actitud de una persona y componente cognoscitivo de la cultura subjetiva que permiten a los individuos realizar categorizaciones necesarias para la toma de decisiones del medio social. ${ }^{22}$ Además, señalan a las condiciones laborales del personal de salud, coincidiendo en este estudio con la categoría "personal de salud", la cual es considerada un hallazgo relevante, debido al papel e influencia del ejercicio del profesional de la salud en el incumplimiento de la vacunación. ${ }^{10}$ Otras razones mencionadas por estos autores, como la edad de la madre y la vivienda, no fueron referidos por las madres entrevistadas en el desarrollo de este estudio.

En la investigación Oportunidades perdidas de vacunación en la población pediátrica se menciona que alguna enfermedad presente en la fecha de aplicación es un factor desencadenante del incumplimiento, ${ }^{23}$ lo que se relaciona dentro de este estudio con el código "estado de salud" perteneciente a la categoría "propias del menor"; es importante mencionar que una contraindicación es una situación en la que no debe administrarse en ninguna circunstancia una vacuna por el elevado riesgo de tener una reacción adversa grave o incluso fatal. Las falsas contraindicaciones no solamente conllevan retrasos innecesarios para la adecuada protección, sino que además favorecen la propagación en la sociedad de creencias erróneas con relación a las situaciones en las que se puede o no vacunar. ${ }^{24}$

\section{Conclusiones}

El incumplimiento del esquema básico de vacunación es un fenómeno multifactorial. A partir de las experiencias compartidas por las madres participantes, se identificó el déficit de conocimiento como un detonante del desinterés hacia la inmunización de los menores debido al desconocimiento de la importancia y el beneficio de la vacunación en la salud de éstos. La educación para la salud es un tema relevante ante esta situación, en este caso, el profesional de salud limitó su ejercicio profesional únicamente a la aplicación del biológico sin mencionar los efectos adversos, la periodicidad con la que hay que acudir, los cuidados que se pudieran requerir y principalmente la importancia de llevarla a cabo. Si se mejorara la interacción entre usuarios y proveedores de servicios de salud en el que se involucre una actitud empática y de profesionalismo, se podría aumentar la oferta de vacunación y estimular su demanda por la población.

Características como la pobreza, la marginación y los conflictos de las comunidades rurales, perjudican en el cumplimiento del esquema básico de vacunación; sin embargo, en este estudio se pudo percibir que en la comunidad rural trabajada esto no influyó de manera trascendente, pues a pesar de ser una comunidad rural que está 
limitada en accesibilidad de servicios de salud, sus habitantes demostraron que han buscado la manera de conseguirlos.

La razón más expresada de este estudio fue el desinterés hacia la vacunación. Dados los resultados encontrados, es importante profundizar en los análisis tanto cualitativos como cuantitativos en otras comunidades y ciudades, para comprender mejor cuáles son las razones y situaciones que influyen en el incumplimiento del esquema básico de vacunación. Tomando en cuenta las razones propias de la madre, del menor, culturales, personal de salud, institucionales, geográficas y económicas, aspectos que aún siguen siendo poco entendidos cuyo conocimiento podría contribuir a la planificación de una estrategia para disminuir este fenómeno.

\section{Bibliografía}

1. Secretaría de Educación Pública. Estrategia Nacional de Atención a la Primera Infancia. [Actualizado en 2019; citado 13 octubre 2020]. Disponible en: http://gaceta.diputados.gob.mx/Gaceta/64/2019/nov/AtPrimeralnfancia.pdf

2. Fondo de las Naciones Unidas para la Infancia. Informe Anual México 2017. [Actualizado en 2018; citado 13 octubre 2020]. Disponible en: https://www.unicef.org.mx/ Informe2017/Informe-Anual-2017.pdf

3. Organización Mundial de la Salud. Estrategia mundial para la salud de la mujer, el niño y el adolescente (2016-2030). [Actualizado en 2015; citado 13 octubre 2020]. Disponible en: https://www.who.int/maternal_child_adolescent/ documents/women-deliver-global-strategy/es/

4. Secretaría de Salud. Programa de Acción Específico Salud para la Infancia y la Adolescencia 2013-2018. [Actualizado en 2019; citado 13 octubre 2020]. Disponible en: http://www.censia.salud.gob.mx/contenidos/descargas/ transparencia/especiales/PAE_Salud_para_la_Infancia_y_ la_Adolescencia.pdf

5. Fondo de las Naciones Unidas para la Infancia. Los derechos de la infancia y la adolescencia en México. [Actualizado 2018; citado 24 de marzo 2020]. Disponible en: https://www.unicef.org/mexico/media/1791/file/SITANUNICEF.pdf

6. Secretaría de Salud. Norma Oficial Mexicana NOM-036SSA2-2012, Prevención y control de enfermedades. Aplicación de vacunas, toxoides, faboterápicos (sueros) e inmunoglobulinas en el humano. Ciudad de México. [Actualizado 28 septiembre 2012; citado 12 octubre 2018] Disponible en: http://dof.gob.mx/nota_detalle.php?codig $0=5270654 \&$ fecha $=28 / 09 / 2012$

7. Secretaría de Salud. Programa de acción especifico vacunación universal 2013-2018. [Internet]. Ciudad de México. [Actualizado en 2013; citado 16 octubre 2018]. Disponible en: http://www.censia.salud.gob.mx/contenidos/descargas/transparencia/especiales/PAE_Vacunacion_Universal_PAE_final_final.pdf

8. Centro de Investigación en Evaluación y Encuestas. Encuesta Nacional de Salud y Nutrición 2018. [Actualizado en 2018; citado 22 de marzo 2020]. Disponible en: https://ensanut. insp.mx/encuestas/ensanut2016/index.php

9. Escobar Díaz F, Osorio Merchán MB, De la Hoz Restrepo F.
Motivos de no vacunación en menores de cinco años en cuatro ciudades colombianas. Rev Panam Salud Publica. 2017; 41(123): 1-6.

10. Escobedo Collado BA, Portocarrero Ramos SL. Factores socioculturales e institucionales relacionados con el incumplimiento del esquema de vacunación en madres de menores de 13 meses. P.S. Peruarbo, Arequipa - 2017. Univ Nac San Agustín. 2018; 4(3): 227-239.

11. Organización Mundial de la salud [internet]. Cobertura vacunal. [Actualizado 15 de julio de 2020; citado 18 de mayo 2020]. Disponible en: https://www.who.int/es/ news-room/fact-sheets/detail/immunization-coverage

12. Consejo Nacional de Evaluación de la Política de Desarrollo Social [internet]. Ciudad de México: CONEVAL; 20052020 [citado 16 mayo 2020]. Disponible en: https:// www.coneval.org.mx/Medicion/Paginas/Glosario.aspx

13. Banco Interamericano de Desarrollo División de Protección Social y Salud. Desarrollo Infantil Temprano en México Diagnóstico y recomendaciones. [Actualizado 2013; citado 13 octubre 2020]. Disponible en: https://publications. iadb.org/es/publicacion/14711/desarrollo-infantil-temprano-en-mexico-diagnostico-y-recomendaciones

14. INEGI. Estadísticas a propósito del día del niño (30 de abril). Comunicado de prensa 201/19; 29 abril 2019; México; Medición de la pobreza: glosario. Disponible en: https:// www.inegi.org.mx/contenidos/saladeprensa/aproposito/2019/nino2019_Nal.pdf

15. Consejo Nacional de Población [internet] México, D.F.: CONAPO. [Actualizado 11 abril 2012; citado 16 mayo 2020]. Localidades rurales. Disponible en: http://www. conapo.gob.mx/es/CONAPO/Localidades rurales

16. Fondo de las Naciones Unidas para la Infancia. Informe anual UNICEF México 2018. [Actualizado en mayo 2019; citado 22 de marzo de 2020]. 55. Disponible en: https:// unicef.org.mx/Informe2018/Informe-Anual-2018.pdf

17. Secretaría de Salud. Atención Primaria De Salud Integral E Integrada Aps-I Mx: La Propuesta Metodológica y Operativa. [Actualizado noviembre 2019; citado 13 octubre 2020]. Disponible en: http://www.sidss.salud.gob.mx/ site2/docs/Distritos_de_Salud_VF.pdf

18. Secretaría de Salud. Manual del Paquete Garantizado de Servicios de Promoción y Prevención para una Mejor Salud. [Actualizado 2011; citado 13 octubre 2020]. Disponible en: https://hospitalcomunitariotarimoro.files. 
wordpress.com/2017/06/manual-del-paquete-garantizado-de-servicios-de-promocic3b3n-y-prevencic3b3n-parauna-mejor-salud.pdf

19. Strauss AL, Corbin JM. Bases de la investigación cualitativa: técnicas y procedimientos para desarrollar la teoría fundada. $1^{\mathrm{a}}$ edición. Colombia: Editorial Universidad de Antioqu?ia; 2002.

20. Ley General de Salud, Secretaría de Salud. Ciudad de México. [Actualizado 24 enero 2020; citado 12 octubre 2018].

21. Scientific Software Development $\mathrm{GmbH}$ [Internet]. Alemania: ATLAS.ti; 2002-2020. [actualizado 2020; citado agosto 2019]. Disponible en: atlas.ti.com

22. Díaz Loving R, Saldívar A, Armenta Hurtarte C, Reyes Nor- ma E, López F, Moreno M. Creencias y Normas en México: Una Actualización del Estudio de las Premisas Psico-Socioculturales. Psykhe (Santiago). 2015; 24(2): 1-25.

23. Alvarado López AD. Oportunidades Perdidas de vacunación en la población pediátrica de 0 a 60 meses que acuden al Centenario Hospital Miguel Hidalgo. Aguascalientes, México: Universidad Autónoma de Aguascalientes; 2014. [tesis]

24. Comité Asesor de Vacunas [Internet]. Madrid: Asociación Española de Pediatría; 1999-2019. [Actualizado enero 2020; citado 24 marzo 2020]. Disponible en: https:// vacunasaep.org/profesionales/contraindicaciones-de-lasvacunas 